\title{
Loneliness Relates to Functional Mobility in Older Adults with Type 2 Diabetes: The Look AHEAD Study
}

\author{
Jeanne M. McCaffery ${ }^{D},{ }^{1}$ Andrea Anderson, ${ }^{2}$ Mace Coday, ${ }^{3}$ Mark A. Espeland, ${ }^{2}$ \\ Amy A. Gorin, ${ }^{4}$ Karen C. Johnson, ${ }^{3}$ William C. Knowler, ${ }^{5}$ Candice A. Myers, ${ }^{6}$ \\ W. Jack Rejeski, ${ }^{7}$ Helmut O. Steinberg, ${ }^{8}$ Andrew Steptoe, ${ }^{9}$ and Rena R. Wing ${ }^{10}$ \\ ${ }^{1}$ Department of Allied Health Sciences, University of Connecticut, Storrs, CT, USA \\ ${ }^{2}$ Department of Biostatistics and Data Science, Wake Forest School of Medicine, Winston-Salem, NC, USA \\ ${ }^{3}$ Department of Preventive Medicine, University of Tennessee Health Science Center, Memphis, TN, USA \\ ${ }^{4}$ Institute for Collaboration on Health, Intervention and Policy, and Psychological Sciences, University of Connecticut, \\ Storrs, CT, USA \\ ${ }^{5}$ Diabetes Epidemiology and Clinical Research Section, Phoenix Epidemiology and Clinical Research Branch, \\ National Institute for Diabetes, Digestive and Kidney Diseases, Phoenix, AZ, USA \\ ${ }^{6}$ Pennington Biomedical Research Center Baton Rouge, Baton Rouge, LA, USA \\ ${ }^{7}$ Department of Health and Exercise Science, Wake Forest School of Medicine, Winston-Salem, NC, USA \\ ${ }^{8}$ Department of Endocrinology, University of Tennessee Health Science Center, Memphis, TN, USA \\ ${ }^{9}$ Behavioural Science and Health Institute of Epidemiology \& Health, University College London, London, UK \\ ${ }^{10}$ Department of Psychiatry and Human Behavior, The Miriam Hospital and Alpert School of Medicine at Brown University, \\ Providence, RI, USA \\ Correspondence should be addressed to Jeanne M. McCaffery; jeanne.mccaffery@uconn.edu
}

Received 10 June 2020; Accepted 19 August 2020; Published 30 October 2020

Academic Editor: F. R. Ferraro

Copyright ( 2020 Jeanne M. McCaffery et al. This is an open access article distributed under the Creative Commons Attribution License, which permits unrestricted use, distribution, and reproduction in any medium, provided the original work is properly cited.

Objective. Little is known about the impact of loneliness on physical health among elderly individuals with diabetes. Here, we examined the relationship of loneliness with disability, objective physical function, and other health outcomes in older individuals with type 2 diabetes and overweight or obesity. Method. Data are drawn from the Look AHEAD study, a diverse cohort of individuals (ages 61-92) with overweight or obesity and type 2 diabetes measured 5-6years after a 10-year weight loss randomized, controlled trial. Results. Loneliness scores were significantly associated with greater disability symptoms and slower 4meter gait speed $(p s<0.01)$. Loneliness did not differ across treatment arms. Discussion. Overall, these results extend prior findings relating loneliness to disability and decreased mobility to older individuals with type 2 diabetes and overweight or obesity.

\section{Introduction}

Loneliness is a subjective state, reflecting a lack of desired closeness with friends, family, and loved ones. Compared with structural measures of social contacts, counting an individual's opportunities for interaction with other people, loneliness assesses the function of social interactions in allowing a person to feel connected to others [1]. Living alone, widowhood, poor health status, and poor functional status each increase risk for loneliness [2, 3]. Roughly, $25-43 \%$ of adults over the age of 70 report being lonely [4].

Loneliness is a well-established correlate of mental health, quality of life [5-7], and early mortality in older adults [8-14]. Moreover, loneliness has previously been shown to relate to disability $[15,16]$ and impaired mobility [17]. For example, loneliness predicted a faster rate of objectively-measured motor decline, defined by motor function and muscle strength, over five years of follow-up among 
985 men and women, with a mean age of 80 [17]. Perissinotto and colleagues [13] found that loneliness was related to greater difficulty with activities of daily living and mobility at six year follow-up among over 14,000 men and women over the age of 60 in the Health and Retirement Study. Higher levels of loneliness also predicted frailty as defined by the Fried Formula at 4 year follow-up among 2,817 individuals over 60 years of age from the English Longitudinal Study of Aging [15]. Interestingly, Hoogendijk and colleagues [18] reported that frailty increased the risk for loneliness over 3 years, suggesting that the relationship between loneliness and physical function may be bidirectional [18].

Little is known about how loneliness relates to health status among older individuals with type 2 diabetes. In the United States, $25 \%$ of individuals over the age of 65 have type 2 diabetes [19] increasing the risk for early mortality, cardiovascular disease, renal disease, dementia, functional impairment, depression, and vision impairment [20]. Although less stringent treatment goals can be recommended for elderly individuals, the need for diabetes self-management remains including treatment adherence, nutrition, and exercise [21]. Social support improves diabetes self-management, medication adherence, diet change, active lifestyles and, in some cases, glycemic control [22]. Conversely, loneliness is associated with less physical activity $[23,24]$ and poorer sleep quality $[25,26]$. As such, it is plausible that loneliness may relate to health outcomes among elderly individuals with type 2 diabetes but these associations have not been established.

Look AHEAD was a randomized controlled trial designed to determine whether 10 years of intensive lifestyle intervention (ILI), comprised of calorie restriction and physical activity promotion to achieve weight loss, improves health outcomes among older individuals with type 2 diabetes and overweight or obesity, relative to a Diabetes Support and Education (DSE) control group. The cohort was reassessed for aging-related outcomes at 15-year follow-up, including loneliness measured for the first time. The goal of this paper is to characterize the prevalence of loneliness among individuals with type 2 diabetes and overweight or obesity in the Look AHEAD cohort and to determine crosssectional associations of loneliness score with self-reported disability and objective mobility and other health indicators, including HbAlc, quality of life, and depressive symptoms. It is hypothesized that loneliness will relate to (1) greater disability and decreased mobility and physical function, as defined by the $400 \mathrm{~m}$ walk, grip strength, and the Short Physical Performance Battery and (2) higher HbAlc and depressive symptoms and lower quality of life.

\section{Methods}

2.1. Research Design. Look AHEAD is a randomized, controlled trial designed to test whether 10 years of ILI, combining calorie restriction and physical activity to produce weight loss, improves health outcomes among individuals with type 2 diabetes and overweight or obesity, relative to DSE [27, 28] (see Supplementary File 2). The cohort was reassessed at year 15 to continue to follow diabetes and aging-related outcomes, including measuring loneliness for the first time. The study enrolled 5,145 men and women, aged $45-76$ at baseline. The present study is cross-sectional and derives variables from 15 year follow-up when participants had a mean age of 75 (range: 61-92). All Look AHEAD participants who were attending clinical visits were included $(n=3187)$. Look AHEAD participants who were followed only through telephone interviews $(n=300)$ were excluded because loneliness was not queried.

2.2. Study Interventions. Eligible patients were randomly assigned to participate in ILI (intervention group) or DSE (comparison group), with stratification according to clinical site. Curricula for the two study groups were developed centrally and have been described in detail previously $[27,28]$.

2.3. Intensive Lifestyle Intervention (ILI). The ILI included calorie restriction, low-fat diet, and increased physical activity and was designed to induce at least a 7\% weight loss at year 1 and to maintain this weight loss in subsequent years. ILI participants were assigned a calorie goal (1200-1800 kcal/d based on initial weight), with less than $30 \%$ of total calories from fat ( $<10 \%$ from saturated fat) and a minimum of $15 \%$ of total calories from protein. The exercise goal was at least 175 minutes of physical activity per week, using activities similar in intensity to brisk walking. ILI participants were seen for 3 groups and one individual session per month for the first 6 months and 2 group, one individual session per month for the next 6 months, and at least monthly through year 10. ILI was effective in inducing and sustaining weight losses relative to the control condition throughout follow-up [28].

2.4. Diabetes Support and Education (DSE). DSE featured three group sessions per year focused on diet, exercise, and social support during years 1 through 4 . In subsequent years, the frequency was reduced to one session annually.

\section{Measures}

3.1. Loneliness. Loneliness was measured using the UCLA Brief Loneliness Scale [29]. The scale contains three questions: "How often do you feel that you lack companionship?", "How often do you feel left out?", and "How often do you feel isolated from others?" Each item has the response choices of "Hardly ever," "Some of the time," and "Often," assigned scores 0,1 , and 2 respectively. These scores for each of the items are summed to give a total score. The prevalence of loneliness has also been defined as reporting "Some of the time" or "Often" relative to "Hardly ever" for at least one of the three questions: "How often do you feel that you lack companionship?", "How often do you feel left out?" and "How often do you feel isolated from others?" [13]. The UCLA Brief Loneliness Scale was shown to have a strong correlation with the full UCLA Loneliness Scale $(r=0.82)$ 
and to have reasonable internal consistency (Cronbach's $\alpha=0.72$ ) [29].

\subsection{Disability and Physical Function}

3.2.1. Pepper Assessment Tool for Disability (PAT-D). The PAT-D is an 18-item self-report questionnaire designed to assess disability in older adults. Participants are asked to rate: "How much difficulty, if any, do you have with each of these activities? Think about the past month. How hard was it to do the activity because of your health?" Items include questions such as "Moving in and out of bed" and "Dressing yourself." Responses range from "Usually did with no difficulty" (1) to "Unable to do" (5) with the possibility of endorsing "Usually did not do for other reasons." Scores are averaged across the 18 items. The PAT-D has shown strong internal consistency $(\alpha=0.82)$ and test-retest reliability $(r>0.70)$.

3.2.2. Physical Function Tests. Objective physical function was assessed in the full cohort at an average of 15-16 year follow-up. The Short Physical Performance Battery Expanded (SPPBexp) [30], a modestly expanded form of the Short Physical Performance Battery [31] designed to minimize ceiling effects of the SPPB when used in well-functioning populations, was administered to assess lower extremity physical function. The SPPB consists of standing balance tasks (side-by-side, semi- and full-tandem stands for 10 seconds each), a $4 \mathrm{~m}$ walk to assess usual gait speed and time to complete five repeated chair stands. The SPPBexp increased the holding time of the standing balance tasks to 30 seconds and added a single leg stand. The SPPBexp component scores are calculated as the ratio of observed performance to the best possible performance and summed to provide a continuous score ranging from 0 to 3 , with higher scores indicative of better performance. Usual walking speed over $20 \mathrm{~m}$ and walking endurance over $400 \mathrm{~m}$ were measured [32]. The course was $20 \mathrm{~m}$ long and marked by cones at each end. Participants were instructed to walk at their usual pace, and time to complete the first $20 \mathrm{~m}$ and the longer $400 \mathrm{~m}$ was recorded. Grip strength $(\mathrm{kg})$ was measured twice in each hand using an isometric Hydraulic Hand Dynamometer (Jamar, Bolingbrook, IL). The maximum force from two trials for the stronger hand was used in the analyses.

\subsection{Other Health Indicators}

3.3.1. Personal Health Questionnaire-9 (PHQ-9). The PHQ9 is a self-administered questionnaire assessing depressed mood and depression severity [33]. The questionnaire asks "How often, over the past two weeks, have you been bothered by any of the following problems?" for nine questions, including "Little interest or pleasure in doing things" and "Feeling down, depressed, or hopeless." Response options include "Not at all," "Several days," "More than half of the days," and "Nearly every day." Depressed mood severity was calculated by assigning response options scores of $0-3$ based on increasing frequency and summing the scores (range: 0-27). The PHQ-9 has strong internal consistency $(\alpha=0.89)$ and test-retest reliability $(r=0.84)$ in clinical samples [33]. The PHQ-9 does not include a loneliness item.

3.3.2. Antidepressant Medications. Participants brought all prescription medications to their annual clinic assessment visits, and these medications (but not the dosages) were recorded by study staff. Antidepressant medications were identified using the Food and Drug Administration classification system.

3.3.3. Quality of Life. Quality of life was assessed using the SF-36 General Health questionnaire [34]. The questionnaire asks participants: "In general, would you say your health is..." with responses ranging from Excellent (1) to Poor (5) on a 1-5 scale. Lower values indicate better general health.

3.3.4. HbA1c and Diabetes Medications. HbA1c was assayed from fasting blood samples. Six major classes of diabetes medications were categorized from the Food and Drug Administration classification system and were used as covariates in analyses of $\mathrm{HbAlc}$.

3.4. Statistical Analysis. Primary analyses were conducted using linear or logistic regression depending on the outcome. Model 1 tested the association of loneliness, age, sex, race, and ethnicity with the function- and health-related variables. Model 2 added depressive symptoms and antidepressant medications to determine whether loneliness relates to the other variables independent of correlated constructs also known to relate to health outcomes. For the relationship of loneliness to $\mathrm{HbA1c}$, the six major categories of diabetes medications were added as covariates to Model 2. Treatment arm was added in Model 3 to determine whether loneliness differs by ILI.

PHQ-9 and PAT-D scales are extremely skewed, even after log transformation, and thus were dichotomized at their lowest value vs anything else.

\section{Results}

4.1. Descriptive Statistics. Descriptive statistics for baseline and the Look AHEAD E visit (15-year follow-up) are presented in Table 1. The balance afforded by the original randomization was maintained at the 15-year visit: no differences in baseline age, sex, race, or Hispanic ethnicity were observed. However, several health indices continued to show intervention effects, including lower BMI ( 32.9 vs 33.6; $p=0.002$ ), faster gait speed (4.85 vs 5.00 seconds; $p=0.01$ ), and less insulin use $(43.4 \%$ vs $49.5 \%$; $p=0.0009)$ in the ILI compared with DSE groups. No differences in loneliness by Look AHEAD treatment arm were observed $(p=0.11)$.

4.2. Prevalence of Loneliness. Thirty-eight percent of the Look AHEAD samples reported being lonely as defined by 
TABLE 1: Characteristics of participants with nonmissing loneliness scale at the Look AHEAD-E visit.

\begin{tabular}{|c|c|c|c|c|c|}
\hline & \multirow{2}{*}{ Nonmissing } & \multirow{2}{*}{ Overall } & \multicolumn{2}{|c|}{ Intervention arm } & \multirow{2}{*}{$p$ value } \\
\hline & & & DSE & ILI & \\
\hline$N$ & & 3190 & 1553 & 1634 & \\
\hline \multicolumn{6}{|l|}{ Baseline characteristics } \\
\hline Age & 3190 & $58.3(6.4)$ & $58.3(6.4)$ & $58.2(6.3)$ & 0.7042 \\
\hline BMI & 3190 & $35.9(6.0)$ & $36.0(5.8)$ & $35.7(6.1)$ & 0.1338 \\
\hline \multicolumn{6}{|l|}{ Gender } \\
\hline Male & 3190 & $1214(38.1 \%)$ & $581(37.4 \%)$ & $633(38.7 \%)$ & 0.4756 \\
\hline Female & & $1976(61.9 \%)$ & $973(62.6 \%)$ & $1003(61.4 \%)$ & \\
\hline \multicolumn{6}{|l|}{ Race/ethnicity } \\
\hline White & & $1939(60.8 \%)$ & $950(61.1 \%)$ & $989(60.5 \%)$ & \\
\hline Black & 3190 & $524(16.4 \%)$ & $260(16.7 \%)$ & $264(16.2 \%)$ & 0.6896 \\
\hline Hispanic & & $440(13.8 \%)$ & $203(13.1 \%)$ & $237(14.5 \%)$ & \\
\hline Others & & $287(9.0 \%)$ & $142(9.1 \%)$ & $145(8.9 \%)$ & \\
\hline \multicolumn{6}{|l|}{ LA-E visit } \\
\hline Age & 3190 & $72.7(6.2)$ & $72.7(6.3)$ & $72.7(6.1)$ & 0.7424 \\
\hline BMI & 3019 & $33.2(6.2)$ & $33.6(6.2)$ & $32.9(6.1)$ & 0.0012 \\
\hline $\mathrm{HbA1c} \%$ & 2665 & $7.5(1.5)$ & $7.5(1.5)$ & $7.5(1.4)$ & 0.5779 \\
\hline PHQ-9 & 3052 & $2.7(3.3)$ & $2.8(3.4)$ & $2.6(3.2)$ & 0.1786 \\
\hline PHQ $-9=0$ & 3052 & $937(30.7 \%)$ & $458(30.8 \%)$ & $479(30.6 \%)$ & 0.9079 \\
\hline SF-36 general health & 3156 & $2.9(0.8)$ & $2.9(0.8)$ & $2.9(0.8)$ & 0.4558 \\
\hline PAT-D & 3157 & $1.5(0.5)$ & $1.5(0.5)$ & $1.5(0.5)$ & 0.5800 \\
\hline PAT-D = 1 & 3157 & $419(13.3 \%)$ & $212(13.8 \%)$ & $207(12.8 \%)$ & 0.4165 \\
\hline $400 \mathrm{~m}$ walk time $(\mathrm{min})$ & 2632 & $6.7(1.9)$ & $6.8(2.0)$ & $6.7(1.9)$ & 0.6073 \\
\hline Gait speed test (sec) & 2949 & $4.93(1.67)$ & $5.00(1.71)$ & $4.85(1.62)$ & 0.0168 \\
\hline Grip strength (right hand) & 2702 & $23.9(9.4)$ & $23.8(9.5)$ & $24.0(9.3)$ & 0.5225 \\
\hline Taking antidepressants & 2822 & $699(24.8 \%)$ & $337(24.5 \%)$ & $362(25.0 \%)$ & 0.7546 \\
\hline Taking any diabetes med & 3067 & $2814(91.8 \%)$ & $1380(92.3 \%)$ & $1434(91.2 \%)$ & 0.2744 \\
\hline Biguanide & 2967 & $2063(69.5 \%)$ & $1008(69.5 \%)$ & $1055(69.6 \%)$ & 0.9868 \\
\hline Insulin & 2894 & $1344(46.4 \%)$ & $704(49.5 \%)$ & $640(43.5 \%)$ & 0.0010 \\
\hline Sulfonylurea & 2863 & $979(34.2 \%)$ & $490(35.1 \%)$ & $489(33.4 \%)$ & 0.3460 \\
\hline TZD & 2758 & $198(7.2 \%)$ & $94(7.0 \%)$ & $104(7.4 \%)$ & 0.6900 \\
\hline Loneliness & 3190 & $3.86(1.38)$ & $3.90(1.42)$ & $3.82(1.34)$ & 0.1164 \\
\hline
\end{tabular}

Values are given as mean (SD) or $N(\%)$

endorsing "Sometimes" to at least on the three questions on the UCLA Brief Loneliness Survey. Nine percent reported "Often" for at least one question.

4.3. Differences in Loneliness Scores by Demographics and BMI. As seen in Supplementary Table 1, levels of loneliness differed meaningfully by demographics and BMI. The mean loneliness score was higher in women compared to men $(p<0.0001)$, individuals of Black, Hispanic, or other races and ethnicities compared to whites $(p=0.0001)$, individuals with less formal education $(p<0.0001)$, and individuals who have a $\mathrm{BMI} \geq 40 \mathrm{~kg} / \mathrm{m}^{2}$ at baseline $(p=0.02)$. A BMI $\geq 40 \mathrm{~kg} / \mathrm{m}^{2}$ at 15 year follow-up also was strongly related to loneliness $(p<0.0001)$, but no differences in loneliness by age were observed at 15 year follow-up $(p=0.38)$.

4.4. Loneliness, Disability, and Mobility. In models adjusting for age, sex, race, and ethnicity (Table 2, Model 1), greater loneliness was significantly related to greater self-report of disability on the Pepper Disability Test $(\mathrm{OR}=1.47$; $p<0.0001)$, slower gait speed $(\beta=0.19 \pm 0.02 ; p<0.0001)$, and weaker hand grip $(\beta=-0.39 \pm 0.10 ; p<0.0001)$.
Loneliness did not correlate with the 400-meter walk test speed $(\beta=0.03 \pm 0.03 ; p=0.2375)$.

After further adjustment for depression symptoms and antidepressant medication use (Table 2, Model 2), greater loneliness continued to significantly relate to higher Pepper Disability Test scores $(\mathrm{OR}=1.24 ; p=0.0018)$ and slower gait $(\beta=0.10 \pm 0.03 ; p=0.0003)$, but the association with grip strength was reduced to nonsignificance $(\beta=-0.17 \pm 0.12$; $p=0.1592$ ). Again, these associations were not substantially altered by statistical control for treatment arm (Table 2, Model 3).

4.5. Loneliness, Depressive Symptoms, Antidepressant Medications, and Quality of Life. In models adjusting for age, sex, race, and ethnicity (Table 2, Model 1), greater loneliness was significantly related to higher $P H Q-9$ scores $(O R=1.89$; $p<0.0001)$ and a greater likelihood of taking antidepressant medications $(\mathrm{OR}=1.32 ; p<0.0001)$. After further adjustment for antidepressant medication use (Table 2, Model 2), greater loneliness continued to relate to higher PHQ-9 scores $(\mathrm{OR}=1.75 ; p<0.0001)$. Similarly, after controlling for PHQ-9 scores, greater loneliness continued to relate to antidepressant use $(\mathrm{OR}=1.12 ; \quad p<0.0001)$. These 
TABLe 2: Adjusted associations with loneliness.

\begin{tabular}{lccccccccc}
\hline \multirow{2}{*}{ Outcome } & \multicolumn{3}{c}{ Model 1 } & & \multicolumn{3}{c}{ Model 2} & \multicolumn{3}{c}{ Model 3 } \\
& Beta & SE & $p$ value & Beta & SE & $p$ value & Beta & SE & $p$ value \\
\hline Gait speed test (seconds) & 0.192 & 0.022 & $<0.0001$ & 0.097 & 0.026 & 0.0002 & 0.096 & 0.026 & 0.0003 \\
Grip strength (right hand) & -0.382 & 0.097 & $<0.0001$ & -0.170 & 0.117 & 0.1471 & -0.168 & 0.118 & 0.1530 \\
400 m walk time & 0.034 & 0.029 & 0.2451 & 0.038 & 0.035 & 0.2759 & 0.037 & 0.035 & 0.2839 \\
SF-36 general health & 0.140 & 0.011 & $<0.0001$ & 0.035 & 0.012 & 0.0057 & 0.035 & 0.013 & 0.0058 \\
HbA1c\% & 0.030 & 0.020 & 0.1372 & 0.009 & 0.023 & 0.6868 & 0.010 & 0.023 & 0.6632 \\
\hline & OR & $95 \%$ CI & $p$ value & OR & $95 \%$ CI & $p$ value & OR & $95 \%$ CI & $p$ value \\
\hline PAT-D $>1$ & 1.470 & $1.313-1.645$ & $<0.0001$ & 1.243 & $1.085-1.424$ & 0.0017 & 1.245 & $1.087-0.427$ & 0.0016 \\
PHQ-9 $>0$ & 1.897 & $1.723-2.088$ & $<0.0001$ & 1.752 & $1.585-1.938$ & $<0.0001$ & 1.754 & $1.586-1.939$ & $<0.0001$ \\
Taking antidepressants & 1.319 & $1.242-1.401$ & $<0.0001$ & 1.126 & $1.048-1.211$ & 0.0013 & 1.127 & $1.048-1.211$ & 0.0012 \\
\hline
\end{tabular}

Note. Model 1: adjusts for age, sex, and race/ethnicity. Model 2: Model 1 plus depressive symptoms (PHQ-9) and antidepressant medication use. Also includes major diabetes med categories for HbAlc outcome (biguanide, insulin, sulfonylurea, and TZD) PHQ-9 outcome does not adjust for depressive symptoms. Taking antidepressants outcome does not adjust for antidepressant medication use. Model 3: Model 2 plus treatment arm.

associations were not substantially altered by statistical control for treatment arm (Table 2, Model 3).

In models adjusting for age, sex, race, and ethnicity (Table 2, Model 1), greater loneliness was significantly related to lower self-rated general health on the SF-36 $(\beta=0.14 \pm 0.01 ; p<0.0001)$, and this association remained significant after controlling for depressive symptoms and antidepressant medication use $(\beta=0.03 \pm 0.01 ; p=0.0063)$ (Table 2, Model 2).

4.6. Loneliness and HbA1c. Loneliness did not correlate with $\mathrm{HbAlc}$ in models adjusted for age, sex, race, and ethnicity ( $\beta=0.03 \pm 0.02 ; p=0.1297)$, nor with further adjustment for depressive symptoms, antidepressant medications, glucoselowering medication, or treatment arm.

4.7. Interaction with Treatment Arm. Treatment arm interacted with loneliness in its association with two mobility measures: 400 -meter walk test $(p=0.03)$ and gait speed $(p=0.03$; Table 3$)$. In each case, physical function was similar across the spectrum of loneliness scores in the ILI, whereas higher loneliness was related to poorer physical function in DSE. Supplementary Figures 1(a) and 1(b) illustrate the interactions with loneliness depicted at the $10^{\text {th }}$ (loneliness $=3$ ) and $90^{\text {th }} \quad$ (loneliness $=6$ ) percentiles of loneliness score.

There was also an interaction of loneliness and treatment arm in their associations with $\mathrm{HbAlc}(p=0.04$, Table 3$)$. In those with lower loneliness scores, there was no differential effect between treatment arms. However, in those with higher loneliness scores, HbAlc was lower in the ILI compared to the DSE (Supplementary Figure 1(c)).

\section{Discussion}

In this sample of older individuals who have type 2 diabetes and overweight or obesity, loneliness was associated with higher disability scores and slower gait speed after statistical adjustment for several potential confounders. Loneliness was also associated with higher depressive symptoms and antidepressant medication use and poorer health-related quality of life after similar adjustment. These results identify loneliness as an important correlate of physical and mental health among aging individuals with an elevated body mass index and type 2 diabetes.

After adjustment for demographic variables, loneliness also correlated with a weaker hand grip but the association weakened to nonsignificance after further adjustment for depressive symptoms and use of antidepressant medications, indicating that loneliness is not independently related to hand grip strength in this study. Loneliness did not significantly relate to HbAlc or the 400-meter walk test in any of the models.

The prevalence of loneliness was similar to prior research in elderly individuals. Roughly $38 \%$ of these individuals with type 2 diabetes and obesity or overweight reported loneliness at least some of the time, and 9\% endorsed experiencing at least one of the loneliness questions often. In the most direct comparison, Perissinotto and colleagues used the UCLA Brief Loneliness Scale (as was used in this paper) to estimate prevalence of loneliness in the Health and Retirement Study, including noninstitutionalized individuals over the age of 65 [13]. Forty-three percent endorsed loneliness on one of the questions at least some of the time, and 13\% reported feeling lonely often, suggesting that the prevalence of loneliness in Look AHEAD is roughly comparable with the U.S. population over the age of 65 .

Levels of loneliness did not differ by randomized treatment arm. This is likely due to the measurement of loneliness 15 years after the initiation of the intervention, and 5 years following the end of the intervention. More research is needed to determine the impact of lifestyle intervention for weight loss on loneliness, particularly in individuals with $\mathrm{BMI} \geq 40$, who showed an increased loneliness scores both at baseline and 15 year follow-up.

Loneliness also appeared to moderate the impact of ILI and DSE on mobility. The interaction indicated that the long-term effect of ILI on gait speed and the $400 \mathrm{~m}$ walk test did not differ by loneliness. However, in DSE, loneliness related to poorer outcomes for gait speed and the $400 \mathrm{~m}$ walk test, leading to treatment arm by loneliness interactions. This suggests that ILI benefited mobility regardless of loneliness level, whereas DSE predicted a lesser overall benefit and the 
TABLE 3: Exploring interactions with loneliness.

\begin{tabular}{|c|c|}
\hline Outcome & $\begin{array}{c}\text { Loneliness by treatment arm } \\
\text { ( } p \text { value })\end{array}$ \\
\hline Gait speed test (seconds) & 0.0325 \\
\hline Grip strength (right hand) & 0.0568 \\
\hline $400 \mathrm{~m}$ walk time & 0.0253 \\
\hline $\mathrm{HbA} 1 \mathrm{c} \%$ & 0.0441 \\
\hline SF-36 general health & 0.2164 \\
\hline PAT-D $>1$ & 0.1308 \\
\hline PHQ-9 $>0$ & 0.2819 \\
\hline Taking antidepressants & 0.8536 \\
\hline
\end{tabular}

benefit varied by loneliness. As DSE had minimal intervention, it is plausible that the relationship of loneliness to mobility in DSE is reflective of prior reports of associations with health outcome in more general populations, e.g., [15-17]. However, the impact of ILI on mobility may have blunted relationships with loneliness.

It further appeared that ILI was more effective in reducing $\mathrm{HbA} 1 \mathrm{c}$ compared with DSE among individuals with higher loneliness, whereas there was little difference by treatment arm among those with lower loneliness levels. It is plausible that ILI may have been more effective among individuals with higher loneliness scores than DSE due to the differential contacts with providers and other participants in ILI. However, it should be recognized that the interaction analyses were exploratory and not hypothesis driven.

Taken together, these findings from a sample of older participants who have type 2 diabetes and overweight or obesity share many similarities to the prior literature involving older people without diabetes. Specifically, we find loneliness to relate to disability scores, objective gait speed, depressive symptoms and use of antidepressant medications, and health-related quality of life, consistent with prior reports $[5,7,13,15-18]$. In contrast, we did not support prior studies finding relationships of loneliness with glycemic control. Given that diabetes impacts one in four elderly adults in the United States, requires a complicated selfmanagement regimen, and portends an increased burden from multiple diseases, it is critical to identify factors that may further compound disease risk.

One challenge of the literature relating loneliness to health outcomes is the variety of different scales used. Metaanalysis and reviews, e.g., $[1,4]$, identify numerous scales designed to characterize loneliness, with some scales showing overlap with structural measures of social connection. Some of the prior research has even relied on a single question to index loneliness. We used the UCLA Brief Loneliness Scale (three items). This scale shows a strong correspondence with the original, 20-item questionnaire [29] and has previously been demonstrated to relate to behavioral and physical outcomes, including depression [7], physical function [16], and mortality [9], among other outcomes. Thus, the scale was a reasonable choice to represent the health impacts of loneliness.
It is important to note that the present study did not measure social isolation, a related but distinct measure of the structure of social contacts. Social isolation also predicts aging-related outcomes [9, 12]. Indeed, several research studies have compared the impact of loneliness to social isolation to determine whether the perception or structure of social contacts has a greater impact on health, but research remains mixed $[1,6,9,15,16,23,35]$. It is also plausible that loneliness and social isolation have synergistic effects wherein those with both conditions are at the greatest health risk [36]. Future research in Look AHEAD should incorporate a measure of social isolation, in addition to loneliness, to determine the relative contributions of each and the potential for compounding risks.

Given the health risks, it is encouraging that interventions are being tested to reduce loneliness in the elderly. For example, Silver Sneakers, a gym membership and exercise classes, was shown to increase physical activity and to reduce social isolation and loneliness compared to matched controls [37]. In addition, a pilot, m-Health intervention targeting maladaptive cognitions in elderly individuals who are experiencing loneliness reduced these symptoms over three months [38].

Although this study had several strengths, including an aging sample with type 2 diabetes, a large sample size, and objective measures of physical function, it is important to note limitations. First, as this study was cross-sectional, the direction of association cannot be determined. Indeed, prior research suggests that the associations of loneliness with depression and disability may be bidirectional $[7,18]$. Future, longitudinal research will be needed to resolve directionality in this cohort. In addition, the loneliness questionnaire was only included in the clinic questionnaire, which excluded $9.4 \%$ of participants who completed telephone interviews but may have had more difficulty attending visits in the clinic. Neither social isolation nor contemporaneous socioeconomic status was measured in Look AHEAD and may serve as confounders of the relationship between loneliness and the health outcomes. Lastly, although we did not see evidence for differential loss of follow-up between treatment groups, we cannot rule out that this may have introduced bias in differences and associations.

Overall, this study demonstrates that loneliness relates to greater disability, slower gait speed, depressive symptoms, antidepressant medication use, and poorer quality of life among older individuals who have type 2 diabetes and are overweight or obese. Future longitudinal research needs to address questions such as the potential for bidirectional relationships with loneliness and the longer-term impacts on health outcomes.

\section{Data Availability}

All Look AHEAD data will be made available through the National Institute of Diabetes, Digestive, and Kidney Disease data repository within two years in accordance with the policy of the Look AHEAD clinical trial. 


\section{Disclosure}

The opinions expressed in this paper are those of the authors and do not necessarily reflect the views of the NIH or other funding sources.

\section{Conflicts of Interest}

All authors report no conflicts of interest.

\section{Acknowledgments}

This study was funded by the National Institutes of Health through cooperative agreements with the National Institute of Diabetes and Digestive and Kidney Diseases (DK57136, DK57149, DK56990, DK57177, DK57171, DK57151, DK57182, DK57131, DK57002, DK57078, DK57154, DK57178, DK57219, DK57008, DK57135, and DK56992). Additional funding was provided by the National Heart, Lung, and Blood Institute; National Institute of Nursing Research; National Center on Minority Health and Health Disparities; NIH Office of Research on Women's Health; and the Centers for Disease Control and Prevention. This research was supported in part by the Intramural Research Program of the National Institute of Diabetes and Digestive and Kidney Diseases. The Indian Health Service (IHS) provided personnel, medical oversight, and facilities. Additional support was received from the Johns Hopkins Medical Institutions Bayview General Clinical Research Center (M01RR02719); the Massachusetts General Hospital Mallinckrodt General Clinical Research Center; the Massachusetts Institute of Technology General Clinical Research Center (M01RR01066); the Harvard Clinical and Translational Science Center (RR025758-04); the University of Colorado Health Sciences Center General Clinical Research Center (M01RR00051); the Clinical Nutrition Research Unit (P30 DK48520); the University of Tennessee at Memphis General Clinical Research Center (M01RR0021140); the University of Pittsburgh General Clinical Research Center (GCRC) (M01RR000056), the Clinical Translational Research Center (CTRC) funded by the Clinical and Translational Science Award (UL1 RR 024153) and NIH grant (DK 046204); the VA Puget Sound Health Care System Medical Research Service, Department of Veterans Affairs; and the Frederic C. Bartter General Clinical Research Center (M01RR01346). The following organizations have committed to make major contributions to Look AHEAD: FedEx Corporation; Health Management Resources; LifeScan, Inc., Johnson and Johnson Company; OPTIFAST ${ }^{\circledR}$ of Nestle HealthCare Nutrition, Inc.; Hoffmann-La Roche Inc.; Abbott Nutrition; and Slim-Fast Brand of Unilever North America. Some of the information contained herein was derived from data provided by the Bureau of Vital Statistics and New York City Department of Health and Mental Hygiene. Details of the Look AHEAD Research group can be found in Supplementary File 2.

\section{Supplementary Materials}

Supplementary Table 1: levels of loneliness by participant characteristics. Supplementary Figure 1(a): interaction between loneliness score and treatment arm in relation to 400 meter walk time. Interaction illustrated using the $10^{\text {th }}$ (loneliness $=3$ ) and $90^{\text {th }}$ (loneliness $=6$ ) percentiles for the loneliness score. Supplementary Figure 1(b): interaction between loneliness score and treatment arm in relation to gait speed. Interaction illustrated using the $10^{\text {th }}$ (loneliness $=3$ ) and $90^{\text {th }}$ (loneliness $=6$ ) percentiles for the loneliness score. Supplementary Figure 1(c): interaction between loneliness score and treatment arm in relation to hemoglobin Alc. Interaction illustrated using the $10^{\text {th }}$ (loneliness $=3$ ) and $90^{\text {th }}$ (loneliness $=6$ ) percentiles for the loneliness score. (Supplementary Materials)

\section{References}

[1] N. Leigh-Hunt, D. Bagguley, K. Bash et al., "An overview of systematic reviews on the public health consequences of social isolation and loneliness," Public Health, vol. 152, pp. 157-171, 2017.

[2] N. Savikko, P. Routasalo, R. S. Tilvis, T. E. Strandberg, and K. H. Pitkälä, "Predictors and subjective causes of loneliness in an aged population," Archives of Gerontology and Geriatrics, vol. 41, no. 3, pp. 223-233, 2005.

[3] J. Cohen-Mansfield, H. Hazan, Y. Lerman, and V. Shalom, "Correlates and predictors of loneliness in older-adults: a review of quantitative results informed by qualitative insights," International Psychogeriatrics, vol. 28, no. 4, pp. 557-576, 2016.

[4] A. D. Ong, B. N. Uchino, and E. Wethington, "Loneliness and health in older adults: a mini-review and synthesis," Gerontology, vol. 62, no. 4, pp. 443-449, 2016.

[5] M. E. Beutel, "Loneliness in the general population: prevalence, determinants and relations to mental health," $B M C$ Psychiatry, vol. 17, no. 1, p. 97, 2017.

[6] J. Golden, R. M. Conroy, I. Bruce et al., "Loneliness, social support networks, mood and wellbeing in communitydwelling elderly," International Journal of Geriatric Psychiatry, vol. 24, no. 7, pp. 694-700, 2009.

[7] J. T. Cacioppo, M. E. Hughes, L. J. Waite, L. C. Hawkley, and R. A. Thisted, "Loneliness as a specific risk factor for depressive symptoms: cross-sectional and longitudinal analyses," Psychology and Aging, vol. 21, no. 1, pp. 140-151, 2006.

[8] R. C. Thurston and L. D. Kubzansky, "Women, loneliness, and incident coronary heart disease," Psychosomatic Medicine, vol. 71, no. 8, pp. 836-842, 2009.

[9] A. Steptoe, A. Shankar, P. Demakakos, and J. Wardle, "Social isolation, loneliness, and all-cause mortality in older men and women," Proceedings of the National Academy of Sciences, vol. 110, no. 15, pp. 5797-5801, 2013.

[10] T. J. Holwerda, A. T. F. Beekman, D. J. H. Deeg et al., "Increased risk of mortality associated with social isolation in older men: only when feeling lonely? Results from the Amsterdam Study of the Elderly (AMSTEL)," Psychological Medicine, vol. 42, no. 4, pp. 843-853, 2012.

[11] R. S. Tilvis, "Suffering from loneliness indicates significant mortality risk of older people," Journal of Aging Research, vol. 2011, p. 534781, 2011.

[12] J. Holt-Lunstad, T. B. Smith, M. Baker, T. Harris, and D. Stephenson, "Loneliness and social isolation as risk factors for mortality," Perspectives on Psychological Science, vol. 10, no. 2, pp. 227-237, 2015.

[13] C. M. Perissinotto, I. Stijacic Cenzer, and K. E. Covinsky, "Loneliness in older persons: a predictor of functional decline 
and death," JAMA Internal Medicine, vol. 172, no. 14, pp. 1078-1083, 2012.

[14] S. Shiovitz-Ezra and L. Ayalon, "Situational versus chronic loneliness as risk factors for all-cause mortality," International Psychogeriatrics, vol. 22, no. 3, pp. 455-462, 2010.

[15] C. R. Gale, L. Westbury, and C. Cooper, "Social isolation and loneliness as risk factors for the progression of frailty: the English Longitudinal Study of Ageing," Age and Ageing, vol. 47, no. 3, pp. 392-397, 2018.

[16] A. Shankar, A. McMunn, P. Demakakos, M. Hamer, and A. Steptoe, "Social isolation and loneliness: prospective associations with functional status in older adults," Health Psychology, vol. 36, no. 2, pp. 179-187, 2017.

[17] A. S. Buchman, "Loneliness and the rate of motor decline in old age: the Rush Memory and Aging Project, a communitybased cohort study," BMC Geriatr, vol. 10, p. 77, 2010.

[18] E. O. Hoogendijk, B. Suanet, E. Dent, D. J. H. Deeg, and M. J. Aartsen, "Adverse effects of frailty on social functioning in older adults: results from the Longitudinal Aging Study Amsterdam," Maturitas, vol. 83, pp. 45-50, 2016.

[19] National Diabetes Statistics Report, Diabetes in Older Adults: A Consensus Report, Centers for Disease Control and Prevention, U.S. Department of Health and Human Services, Atlanta, GA, USA, 2017.

[20] M. S. Kirkman, V. J. Briscoe, N. Clark et al., "Diabetes in older adults: a consensus report," Journal of the American Geriatrics Society, vol. 60, no. 12, pp. 2342-2356, 2012.

[21] American Diabetes Association, "Standards of medical care in diabetes," Diabetes Care, vol. 42, no. 1, 2019.

[22] J. L. Strom and L. E. Egede, "The impact of social support on outcomes in adult patients with type 2 diabetes: a systematic review," Current Diabetes Reports, vol. 12, no. 6, pp. 769-781, 2012.

[23] A. Shankar, A. McMunn, J. Banks, and A. Steptoe, "Loneliness, social isolation, and behavioral and biological health indicators in older adults," Health Psychology, vol. 30, no. 4, pp. 377-385, 2011.

[24] L. C. Hawkley, R. A. Thisted, and J. T. Cacioppo, "Loneliness predicts reduced physical activity: cross-sectional \& longitudinal analyses," Health Psychology, vol. 28, no. 3, pp. 354-363, 2009.

[25] B. Yu, A. Steptoe, K. Niu, P.-W. Ku, and L.-J. Chen, "Prospective associations of social isolation and loneliness with poor sleep quality in older adults," Quality of Life Research, vol. 27, no. 3, pp. 683-691, 2018.

[26] L. M. Kurina, K. L. Knutson, L. C. Hawkley, J. T. Cacioppo, D. S. Lauderdale, and C. Ober, "Loneliness is associated with sleep fragmentation in a communal society," Sleep, vol. 34, no. 11, pp. 1519-1526, 2011.

[27] D. H. Ryan, M. A. Espeland, G. D. Foster et al., "Look AHEAD (Action for Health in Diabetes): design and methods for a clinical trial of weight loss for the prevention of cardiovascular disease in type 2 diabetes," Controlled Clinical Trials, vol. 24, no. 5, pp. 610-628, 2003.

[28] The Look AHEAD Research Group, "Cardiovascular effects of intensive lifestyle intervention in type 2 diabetes," New England Journal of Medicine, vol. 369, pp. 145-154, 2013.

[29] M. E. Hughes, L. J. Waite, L. C. Hawkley, and J. T. Cacioppo, "A short scale for measuring loneliness in large surveys," Research on Aging, vol. 26, no. 6, pp. 655-672, 2004.

[30] J. M. Guralnik, E. M. Simonsick, L. Ferrucci et al., "A short physical performance battery assessing lower extremity function: association with self-reported disability and prediction of mortality and nursing home admission," Journal of Gerontology, vol. 49, no. 2, pp. M85-M94, 1994.

[31] E. M. Simonsick, A. B. Newman, M. C. Nevitt et al., "Measuring higher level physical function in well-functioning older adults: expanding familiar approaches in the Health $\mathrm{ABC}$ study," The Journals of Gerontology Series A: Biological Sciences and Medical Sciences, vol. 56, no. 10, pp. M644-M649, 2001.

[32] E. M. Simonsick, P. S. Montgomery, A. B. Newman, D. C. Bauer, and T. Harris, "Measuring fitness in healthy older adults: the health ABC long distance corridor walk," Journal of the American Geriatrics Society, vol. 49, no. 11, pp. 1544-1548, 2001.

[33] K. Kroenke, R. L. Spitzer, and J. B. W. Williams, “The PHQ-9," Journal of General Internal Medicine, vol. 16, no. 9, pp. 606-613, 2001.

[34] J. E. Brazier, R. Harper, N. M. Jones et al., "Validating the SF36 health survey questionnaire: new outcome measure for primary care," BMJ, vol. 305, no. 6846, pp. 160-164, 1992.

[35] J. Beller and A. Wagner, "Disentangling loneliness: differential effects of subjective loneliness, network quality, network size, and living alone on physical, mental, and cognitive health," Journal of Aging and Health, vol. 30, no. 4, pp. 521-539, 2018.

[36] J. Beller and A. Wagner, "Loneliness, social isolation, their synergistic interaction, and mortality," Health Psychology, vol. 37, no. 9, pp. 808-813, 2018.

[37] S. Brady, "Reducing isolation and loneliness through membership in a fitness program for older adults: implications for health," Journal of Applied Gerontology, vol. 37, 2018.

[38] M. A. Jarvis, A. Padmanabhanunni, and J. Chipps, "An evaluation of a low-intensity cognitive behavioral therapy mHealth-supported intervention to reduce loneliness in older people," International Journal of Environmental Research and Public Health, vol. 16, no. 7, 2019. 